\title{
Phase Contrast Cryo-Electron Tomography and Single Particle Analysis with a New Phase Plate.
}

\author{
Maryam Khoshouei ${ }^{1}$, Radostin Danev ${ }^{1}$, Günther Gerisch ${ }^{2}$, Maria Ecke ${ }^{2}$, Juergen Plitzko ${ }^{1}$, Wolfgang \\ Baumeister $^{1}$
}

1. Max Planck Institute of Biochemistry, Department of Molecular Structural Biology, Martinsried, 82152, Germany.

2. Max Planck Institute of Biochemistry, Department of Cell Dynamics, Martinsried, 82152, Germany

Biological samples are very sensitive to beam irradiation because of induced changes to their structure by the incident electron. Due to the limited allowed dose, cryo-images of frozen-hydrated biological specimens are typically noisy and low in contrast. One way to improve the imaging of such samples is by using a phase plate to increase contrast and signal-to-noise ratio. The improved contrast comes from the phase shift that the phase plate introduces between the scattered electron beams and the unscattered electron beams.

The phase plate is located on the back-focal plane of the TEM. It changes the sine type CTF to a cosine type therefore; the object information in the low spatial frequency domain is transferred well into the image. In contrast, the conventional defocus phase contrast TEM suppresses low-frequency information due to its sine type CTF [1].

There are many types of phase plates e.g. thin film, electrostatic and magnetic type. However, there have been some technical difficulties in designing and fabrication of the electrostatic and the magnetic phase plates. The most successful phase plate has been thin carbon film Zenrike phase plate [2]. Recently, we developed a new type of the phase plate which has much longer life time and overall better performance.

The aim of this research is development and applications of phase contrast method being carried out at Max-Planck Institute of Biochemistry in Germany in collaboration with FEI in the Netherlands.

The structure of a whole vitrified earth worm sperm have been resolved by cryo-electron tomography with a new generation of phase plate. The worm sperm is comprised of acrosomal complex, nucleus, mitochondria and flagellum. We have obtained 3D structural information on the different parts with a complete overlap. The flagellum consists of a central microtubule pair surrounded by nine doublet microtubules. Glycogen granules are positioned around the microtubule doublet. The whole cell structure is enveloped by a plasma membrane. Previous work, both in cryo and plastic, identified various molecular complexes of the flagellum. Here, we show the superior results achieved by phase plate tomography [3].

Furthermore, the performance of the new phase plate has been tested for single particle analysis of $\beta$ galactosidase. In both cryo-electron tomography and single particle analysis contrast improvement with no fringe artefacts was observed. 


\section{References:}

[1] R. Danev, S. Kanamaru, M. Marko and K. Nagayama, Journal of Structural Biology 171 (2010), p. 174-181.

[2] R. Danev and K. Nagayama, Journal of Structural Biology 161 (2010), p. 211-218. [3] A.

Rolando et al, International Journal of Morphology (2007), p. 277-284
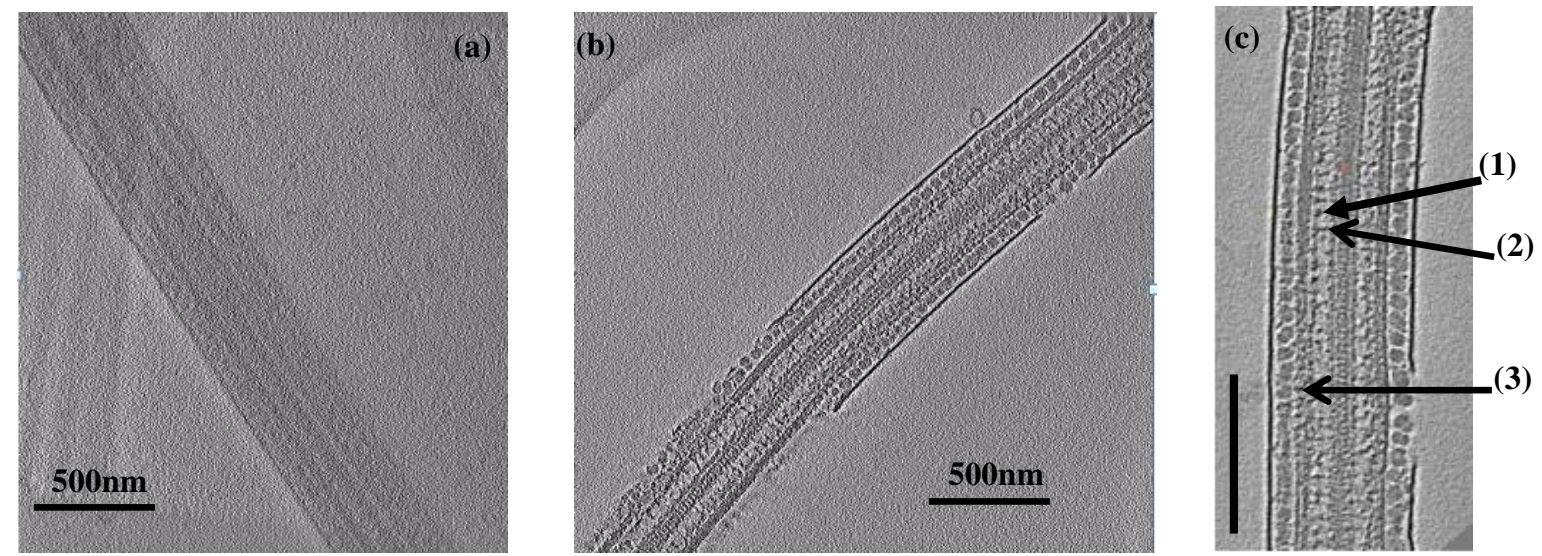

Figure 1. Slices of tomograms without (a) \& with (b) phase plate. Visibility of radial spokes (2), inner dynein (1) and outer dynein (3) from a single tomogram (scale bar: 500nm) (c). [Titan Krios 300kV, energy filter, direct detector, defocus: -500nm, Magnification: 26000].

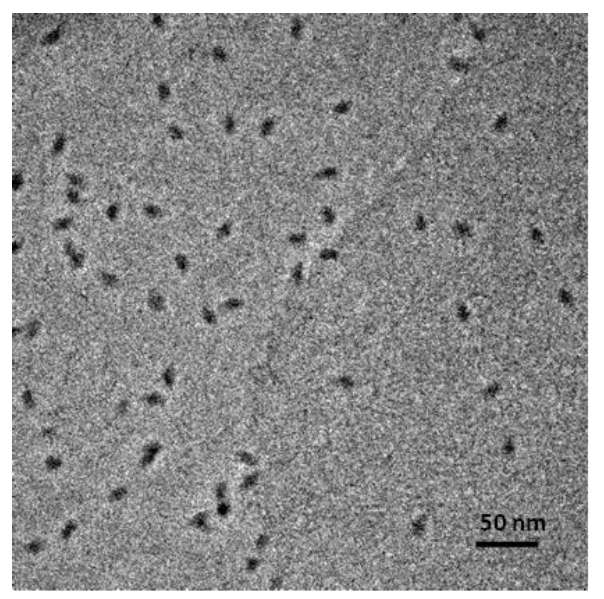

Figure 2. Single particle analysis of $\beta$ galactosidase, Tecnai F20, defocus:-200nm, Magnification: 89000 